\title{
Is parasite clearance clinically important after malaria treatment in a high transmission area? A 3-month follow-up of home-based management with herbal medicine or ACT
}

\author{
Merlin L. Willcox ${ }^{\mathrm{a}, \mathrm{b}, \mathrm{c}, *}$, Bertrand Graz ${ }^{\mathrm{a}, \mathrm{c}, \mathrm{d}, \mathrm{e}}$, Chiaka Diakite ${ }^{\mathrm{c}, \mathrm{g}}$, Jacques Falquet ${ }^{\mathrm{a}, \mathrm{c}}$, \\ Florent Dackouo ${ }^{\mathrm{f}}$, Oumar Sidibe ${ }^{\mathrm{c}, \mathrm{g}}$, Sergio Giani ${ }^{\mathrm{a}, \mathrm{c}, \mathrm{h}}$, Drissa Diallo ${ }^{\mathrm{g}}$ \\ a Antenna Technologies, Geneva, Switzerland \\ b Department of Primary Health Care, University of Oxford, Oxford, UK \\ c Research Initiative for Traditional Antimalarial Methods, Oxford, UK \\ d Institute of Social and Preventive Medicine (IUMSP), University of Lausanne, Switzerland \\ e Institute of Social and Preventive Medicine (IMSP), University of Geneva, Switzerland \\ ${ }^{\mathrm{f}}$ Faculté de Médecine, Pharmacie et Odonto-Stomatologie, Université de Bamako, Bamako, Mali \\ g Département de Médecine Traditionnelle, INRSP, Bamako, Mali \\ h Aidemet NGO, Bamako, Mali
}

\section{A R T I C L E I N F O}

\section{Article history:}

Received 4 March 2010

Received in revised form 4 October 2010

Accepted 4 October 2010

Available online 5 November 2010

\section{Keywords:}

Malaria

Home-based management

Herbal medicine

Argemone mexicana

Artemisinin Combination Therapy

Randomised Controlled Trial

\begin{abstract}
A B S T R A C T
Argemone mexicana (AM), a validated herbal medicine for uncomplicated malaria, seems to prevent severe malaria without completely clearing parasites in most patients. This study, in a high transmission area of South Mali, explores whether residual parasitaemia at day 28 was associated with subsequent malaria episodes and/or anaemia.

Three hundred and one patients were randomly assigned to AM or artesunate/amodiaquine as first line treatment, of whom 294 were followed up beyond the standard 28 days, to 84 days. From day 29 to day 84, there were no significant differences between treatment groups in new clinical episodes of uncomplicated malaria ( 0.33 vs 0.31 episodes/patient), severe malaria ( $<6 \%$ per month of patients aged $\leq 5$ years) or moderate anaemia (hematocrit $<24 \%: 1.1 \%$ in both groups at day 84 ). Total parasite clearance at day 28 was not correlated with incidence of uncomplicated or severe malaria or of moderate anaemia over the subsequent two months.

Total parasite clearance at day 28 was not clinically important in the context of high transmission. If this finding can be confirmed, some antimalarials which are clinically effective but do not completely clear parasites could nevertheless be appropriate in high transmission areas. Such a policy could be tested as a way to delay resistance to artemisinin combination therapies.
\end{abstract}

(C) 2010 Royal Society of Tropical Medicine and Hygiene. Published by Elsevier Ltd. All rights reserved.

\section{Introduction}

Malaria is one of the principle causes of mortality in African children, and one of the main causes of morbidity in tropical countries. Although effective treatments

\footnotetext{
* Corresponding author. Tel.: +44 1865 289288; fax: +44 1865289287.

E-mail address: merlin.willcox@dphpc.ox.ac.uk (M.L. Willcox).
}

exist, their widespread use for the home-based management of malaria (HMM) would favour the spread of drug resistance. ${ }^{1}$ Many populations in remote areas rely on local traditional herbal remedies for the first-line treatment of malaria. $^{2}$

Local communities in Mali use herbal preparations as the first treatment for more than $80 \%$ of malaria episodes. ${ }^{3}$ Argemone mexicana (AM) decoction was selected as the most promising from 160 traditional recipes. ${ }^{3-5}$ This 
plant is all the more interesting because it is widespread throughout the tropics (including Latin America and Asia). After completion of toxicological studies, a dose-escalating clinical study showed a dose response, no serious adverse events and a sharp decline in parasitaemia (from a geometric mean of 14859 at day 0 to a geometric mean of 174 parasites per mcl at day 4) although generally without total parasite clearance. ${ }^{6}$

Two strategies for HMM were compared in a randomised controlled trial (RCT): AM as first line treatment with artesunate/amodiaquine (As/Aq) as second line ('AM group'); or the standard As/Aq as first line with another artemisinin combination therapy (ACT), arthemeter/lumefantrine, as second line ('ACT group'). ${ }^{7}$ Following the usual practice of assessing antimalarial treatments by outcome in the 28 days after diagnosis, we found that progress to severe malaria was $1.9 \%$ in children aged $\leq 5$ years in both groups, and $0 \%$ among those aged $>5$ years old, so that the incidence of severe malaria was kept to a lower level than reported in comparable studies of HMM. ${ }^{8}$ However the AM and ACT groups were very different in terms of parasite clearance. The proportion of patients with parasitaemia at day 28 was $63-76 \%$ in the AM group and $21-49 \%$ in the ACT group (lower and upper estimates, $P<$ 0.001 with both estimates).

There has been some debate on the clinical significance of total parasite clearance, especially in areas of high transmission. WHO regards parasite clearance at 28 days as the gold standard for evaluating drug efficacy. ${ }^{9}$ After parasite clearance in asymptomatic patients, the incidence of clinical malaria episodes was reduced for 56 days, but then increased from days $56-112 .^{10}$ This suggests that patients are re-infected within a few weeks.

As our hypothesis was that the AM and ACT treatment strategies could be clinically equivalent (hence a 'noninferiority trial' design) but differ in parasite clearance at 28 days, we decided to follow up the patients in the RCT for an extended period of 84 days, in order to detect potential differences between the two groups after the conventional 28 day study. In order to produce results useful for policy makers, we focused on outcome measures of public health importance: incidence of uncomplicated and severe malaria episodes; prevalence of anaemia; serious adverse events; re-treatments with the second-line antimalarial and cost. Malian medical and public health authorities were interested in a real situation estimate (at least 'as real as possible'), the primary aim being to study a new 'médicament traditionnel amélioré' (Improved Traditional Drug), with a reasonable estimate of the effects to be anticipated when used outside of clinical settings.

\section{Methods}

\subsection{Participants}

This study was conducted in the remote village of Missidougou, in the south-east of Mali. Patients of all ages first consulted the village health worker, who referred to the study team all patients whom he presumed to have malaria (mainly on the basis of a history of fever). Pregnant women were included since AM decoction is regularly used by pregnant women, and animal studies showed no evidence of reproductive toxicity. We included all consenting patients considered as having malaria by the village health worker (in order to reflect the real field situation of public health programmes), with a history of fever in the last day, except those with signs of severe malaria, those who had already ingested a full dose of antimalarials on the same day, and those unable to return for follow-up.

\subsection{Treatment interventions}

The treatment interventions, randomisation and follow-up in the first 28 days have been described elsewhere. ${ }^{7}$ In summary, sample size was computed in order to detect a difference of $15 \%$ assuming an adequate clinical response rate of at least $85 \%$ with an ACT $(\alpha=$ $0.05,1-\beta=0.80$, two-tailed test). Patients were randomly assigned to two groups, AM or ACT, using a computergenerated random number table and a 2:1 randomisation ratio with blocks of six stratified for age ( $<1$ year, 1-5 years, $>5$ years). Due to the very different nature of the two treatments, it was not possible to blind the patients or the clinicians to the treatments being taken. However the treatment groups were not known to the microscopists when performing parasite counts.

The first-line treatment (detailed below) was used for the initial illness, and during the follow-up period for any new febrile episode in a patient who had previously improved more than 14 days after the start of the previous treatment. Blood films were taken before the start of treatment but were analysed later. The second-line treatment was used only if the first-line treatment failed or if there were signs of severity. In both groups intramuscular artemether was used if the patient was unable to swallow. If there were signs of another infection (for example a chest infection) this was also treated.

AM Group: AM aerial parts were harvested and dried. For each new treatment, patients (or their parents) were given a bag of $500 \mathrm{~g}$ of the dried plant material, and were told how to prepare the decoction. Patients were advised to take this treatment for one to two weeks. The second-line treatment was oral As/Aq.

ACT Group: Patients were given artesunate and amodiaquine tablets in a co-blister pack (Arsucam ${ }^{\circledR}$, Sanofi-synthelabo), according to their weight, in the dosage schedule given on the pack insert. Women who were known to be pregnant were treated with sulphadoxinepyrimethamine (SP), three tablets in a single dose, instead of As/Aq. The second-line treatment was oral artemether/lumefantrine (Coartem ${ }^{\circledR}$, Novartis).

\subsection{Outcomes}

The primary outcome measure for comparison over the 84-day follow-up was incidence of new episodes of uncomplicated malaria (fever reported in the last 24 hours, irrespective of other infections, confirmed on thick film).

Secondary outcome measures were incidence of severe malaria from days 29 to 84, prevalence of moderate anaemia (haematocrit $<24 \%$ ) at day 84 , incidence of adverse events after day 28 (including follow-up of the babies 
of women pregnant at the time of treatment), need for retreatment, and cost per patient over the 84 days of follow-up. Cost was calculated on the basis of wholesale costs of medications in Mali at the time of the study, and hospital bills for any referred patients. The herbal medicine had no monetary cost as it was harvested and prepared locally. In the cost analysis, we included all treatment costs relating to episodes of presumed malaria (cost of drugs, ancillary treatments, and hospital admission). We did not include opportunity costs of the patients' and their families' time as this would have been difficult to estimate in a community of subsistence farmers where most people do not receive a salary.

\subsection{Follow-up}

Patients were followed up on days 56 and 84, and were advised to return at any time if they experienced a deterioration of their condition, or recurrence of symptoms. Patients who did not return for follow-up were visited at home. Thin and thick blood films were taken at baseline and every follow-up visit. Haematocrit was taken at baseline, on days 28, 56 and 84, and in cases of treatment failure. A follow-up visit was made seven months after the start of the trial to all women pregnant during the study, and a standard screening examination was performed to look for any abnormalities in their babies.

Laboratory methods are described in detail elsewhere. ${ }^{7}$ All blood films taken on d 0, d 7, d 28 and the day of a presumed new episode of malaria were read independently by two microscopists. Parasites were counted per 200 leucocytes (and per 500 leucocytes if there were fewer than 10 parasites per 200 leucocytes). For the purpose of calculating parasitaemia the leucocyte count was assumed to be $7.5 \times 10^{9} / \mathrm{mcl}$. A slide was not declared negative until 100 high power fields had been examined. A sample of discordant slides was read by a third microscopist. All three microscopists were experienced so discordant positive/negative results were explored through sensitivity analysis rather than assuming that one microscopist was consistently 'correct'. The parasitaemia threshold for confirming a new episode of malaria was set at $\geq 100$ per microlitre because very low parasitaemia was deemed unlikely to cause febrile illness in a semi-immune population, and because at very low parasitaemia there were often discrepancies between microscopists as to the presence or absence of parasites. Total parasite clearance was defined as no parasites seen by either microscopist. Haematocrit was measured using microhaematocrit tubes in a centrifuge. Some haematocrit values are missing because some of the capillary tubes burst open in the centrifuge.

\subsection{Statistical analysis}

Results were analysed by intention to treat. Patients were only excluded in the course of the study if they withdrew consent or if they took a course of antimalarial medication which had not been prescribed by the study team. Data were entered and analysed using Epi-info 6 software (CDC, Atlanta, GA, USA), with $\chi^{2}$ and Fisher's exact tests for discrete variables, and Kruskall-Wallis test for means. We stratified by age group $(<1,1-5,>5)$ for the analysis of the association between parasite clearance at day 28 and the incidence of recurrent malaria. We used logistic regression (SPSS version 16.0, SPSS Inc., Chicago, IL, USA) to adjust for the effect of multiple variables: age, sex, temperature at day 28 , parasite count at day 28 , and haematocrit at day 28 . As the distribution of parasite counts was skewed we used the following categories of parasite counts: 0, 1-99, 100-1999, 2000-15000, and >15 000.

\subsection{Ethical issues}

Patients were only included if they (or in the case of children, their parents) gave written informed consent. Patients were followed closely, and were given alternative treatment if necessary. All treatments were provided free of charge, including referral to hospital when necessary.

\section{Results}

\subsection{Patients included}

Recruitment took place from 29 July to 8 September 2006. Three hundred and one patients were included, and participant flow is summarised in Figure 1.

\subsection{Baseline characteristics}

Baseline characteristics are summarised in Table 1. Of those with a positive blood film, only six (2\%) did not have a pure Plasmodium falciparum infection.

\subsection{Compliance}

All patients in the AM group reported that they drank the decoction and only 5 patients did not wash with it; 88 patients took the treatment for more than 7 days, although only 47 of these had been advised to do so by the study team. Only 4 (2\%) took less than the recommended dose. In contrast $97 \%$ of patients in the ACT group reported compliance with the treatment (the two cases who did not comply with instructions took a double dose on day 0 ).

\subsection{Outcomes at day 28}

The outcomes at 28 days were reported in detail elsewhere. ${ }^{7}$ In summary, incidence of severe malaria was under 2.5\% in both groups. Most of these cases involved severe anaemia. There were no cases of coma or convulsions, and no deaths. Clinical cure rates (defined as clearance of fever and other malaria symptoms by day 14) were similar. Six percent of the AM group and $5 \%$ of the ACT group had to be retreated with the second-line antimalarial by day 14 . A new episode of malaria occurred in $16 \%$ of the AM group and $11 \%$ of the ACT group between days 15 and 28. The incidence of adverse events was similar in both groups (14\% and 19\%), and no serious adverse events were observed. 


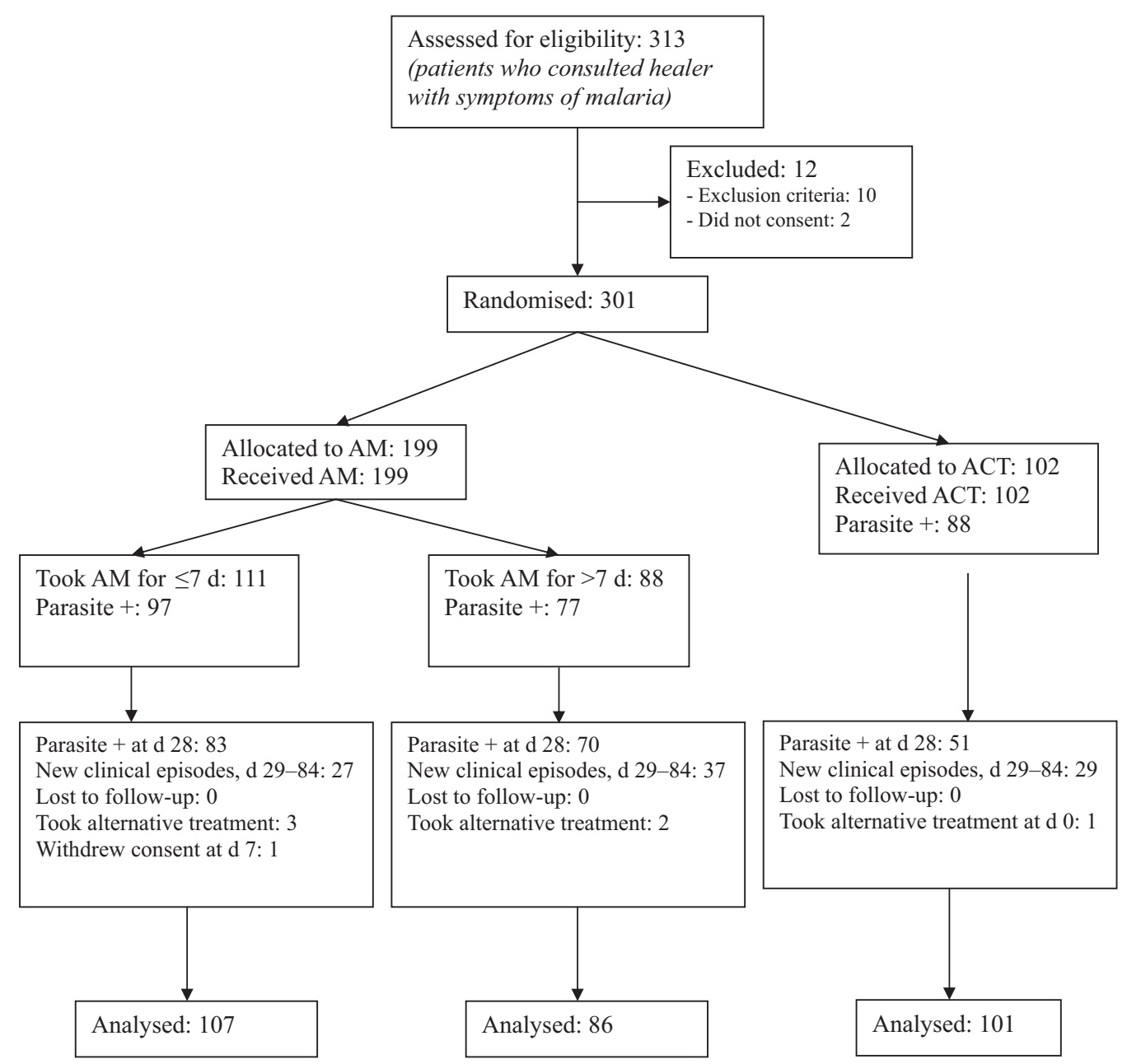

Figure 1. Flowchart of patients. ( $\mathrm{AM}=$ Argemone mexicana decoction as first-line; $\mathrm{ACT}=$ artesunate $/$ amodiaquine as first line $).$

\subsection{Outcomes at day 84 (Table 2)}

At day 84 , seven patients were excluded, one who had withdrawn consent at day 7 and six who had taken an antimalarial from another source (which may have affected the outcomes).

\subsection{Incidence of uncomplicated malaria, days 29-84}

There were 102 episodes treated presumptively as new uncomplicated malaria in the AM group ( 0.53 per patient) and 50 in the ACT group ( 0.50 per patient, $P=0.65)$.
On the day of these new episodes, blood films were taken in 97 and 50 in the AM and ACT groups respectively. Of those who had blood films taken, uncomplicated malaria was confirmed with parasitaemia $\geq 100 / \mathrm{mcl}$ in 63 (0.33 per patient) and 31 (0.31 per patient) in the AM and ACT groups respectively $(P=0.76)$. Of these, seven episodes were treated presumptively as severe malaria although subsequent analysis confirmed that they fulfilled the criteria for uncomplicated malaria. Within the AM group, subgroup analysis showed that the number of new episodes was significantly greater in the patients who had taken the decoction for more than seven days ( 0.435 per

Table 1

Baseline characteristics of included patients.

\begin{tabular}{|c|c|c|}
\hline Group & $\mathrm{AM}(n=193)$ & $\mathrm{ACT}(n=101)$ \\
\hline Males, $n(\%)$ & $94(48.7)$ & $43(42.6)$ \\
\hline Median age, years (IQR) & $5(2-20)$ & $5(2-20)$ \\
\hline Mean temperature, ${ }^{\circ} \mathrm{C}(\mathrm{SD})$ & $37.0(1.3)$ & $37.1(1.4)$ \\
\hline Thick film positive, $n(\%)$ & $171(88.6)$ & $87(86.1)$ \\
\hline Geometric mean parasite count in patients with positive thick film (per mcl) & 908 & 975 \\
\hline Haematocrit, \% (SD) [ $n$ tested] & $34.3(6.0)[153]$ & $34.8(6.0)[72]$ \\
\hline
\end{tabular}

AM: Argemone mexicana group; ACT: Artemisinin Combination Therapy group. 
Table 2

Outcome measures over months 2 and 3 (days 29 to 84 ) according to treatment group [95\% CI].

\begin{tabular}{|c|c|c|c|}
\hline Treatment group & $\mathrm{AM}(n=193)$ & $\mathrm{ACT}(n=101)$ & $P$-value \\
\hline Incidence per patient of uncomplicated malaria (confirmed) & $0.33[0.26-0.40]$ & $0.31[0.22-0.41]$ & 0.35 \\
\hline Age-specific Incidence of severe malaria, in patients aged $\leq 5$ years & $5 / 102(4.9 \%)[1.6-11.1]$ & $1 / 52(1.9 \%)[0.05-10.3]$ & 0.37 \\
\hline Prevalence of moderate anaemia at day $84, n(\%)$ & $2(1.1)$ & $1(1.1)$ & 0.98 \\
\hline
\end{tabular}

AM: Argemone mexicana group; ACT: Artemisinin Combination Therapy group. There was no severe malaria among patients $>5 y e a r s$ old.

patient) compared to those who had not (0.262 per patient, $P=0.011)$.

\subsection{Incidence of severe malaria, day $29-84$}

All cases of severe malaria occurred in children aged 4 years or younger. We calculated the incidence for the age group $\leq 5$ years: there were five episodes in 102 children in the AM group (incidence $4.9 \%$ over 2 months) compared to one in 52 children in the ACT group (1.9\% over 2 months, $P=0.37)$. In each group, $1.9 \%$ of patients had coma/convulsions, and $1.9 \%$ in the AM group developed severe anaemia (haematocrit $<15 \%$ ) requiring a transfusion. One patient in the AM group had a second episode of severe malaria with persistent vomiting (at day 49, following treatment with $\mathrm{i}-\mathrm{m}$ artemether for persistent vomiting at d 14). One patient (a one year old girl) died suddenly at home following a convulsion at day 44 , having previously made a good recovery after being given the second-line treatment (As/Aq) at day 14. A thick film taken on day 28 (but analysed later) showed a parasitaemia of $2645 / \mathrm{mcl}$, at which time she was asymptomatic. She did not attend again before her death. It is probable that the fatal illness was severe malaria 30 days after treatment with As/Aq.

\subsection{Anaemia}

The prevalence of moderate anaemia (haematocrit $<24 \%$ ) was the same in both groups at d 84 (measured in $183 / 193$ and $94 / 101$ of the AM and ACT groups respectively).

\subsection{Parasitaemia}

In patients who were parasite positive at baseline (Figure 2), parasites had been cleared by day 7 in $22.6 \%$ of the AM group and $77.5 \%$ in the ACT group $(P<0.0001)$, but by day 28 , only $17.9 \%$ and $50.0 \%$ remained clear of parasites in each group respectively $(P<0.0001)$. By day 84 , the difference between the groups was further reduced $(29.2 \%$ and $41.4 \%$ free of parasites in the AM and ACT groups respectively, $P=0.05$ ). In both groups geometric mean parasitaemia was markedly reduced compared to the levels at baseline over the length of follow-up (below 200 parasites/mcl in both groups, see Figure 3), and there was no significant difference in incidence of clinical episodes of malaria between the groups. In those who were parasite negative at baseline (Figure 4), 56\% in the AM group became parasitaemic by day 14 , compared to $7 \%$ in the ACT group $(P=0.003)$ but by day 28 , $60 \%$ and $57 \%$ were parasitaemic in the AM and ACT groups respectively $(P=0.49)$. Again there were no differences

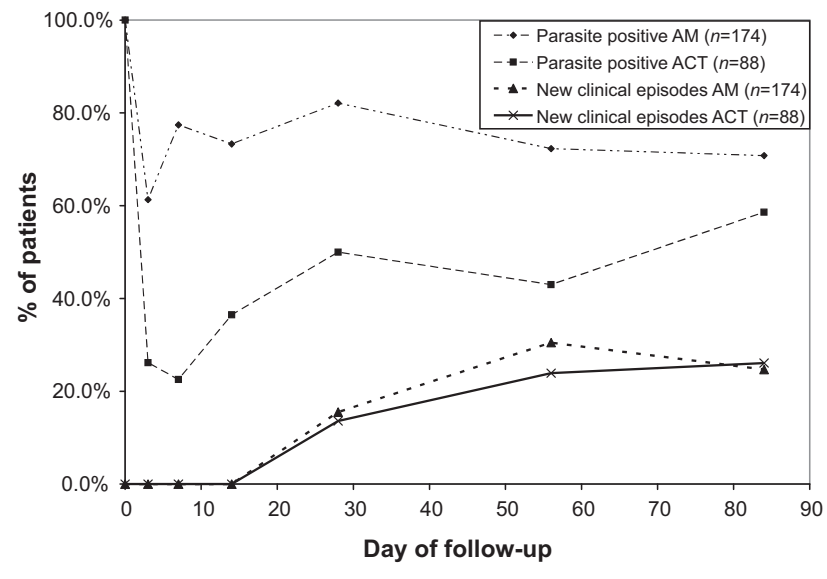

Figure 2. Parasite clearance and new clinical episodes of malaria in patients with a positive malaria film at baseline (AM = Argemone mexicana group, $\mathrm{ACT}=$ Artemisinin Combination Therapy group). The 'parasite positive' lines show the percentage of patients who had parasites on thick blood films at the chosen timepoints. The 'new clinical episodes' lines show the percentage of patients who experienced a new episode of clinical malaria since the last scheduled follow-up visit.

in incidence of clinical episodes of malaria between the groups.

\subsection{The importance of parasite clearance}

An exploratory analysis compared patients with and without total parasite clearance at day 28 (irrespective of treatment group). We compared the incidence of uncomplicated and severe malaria from days 29-84, and

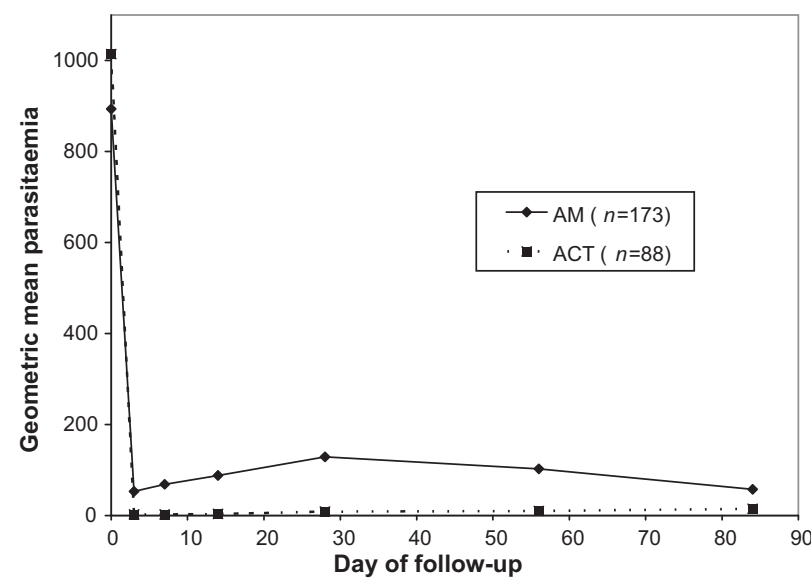

Figure 3. Geometric mean parasitaemia for patients with a positive malaria film at baseline $(\mathrm{AM}=$ Argemone mexicana group, $\mathrm{ACT}=$ Artemisinin Combination Therapy group). 


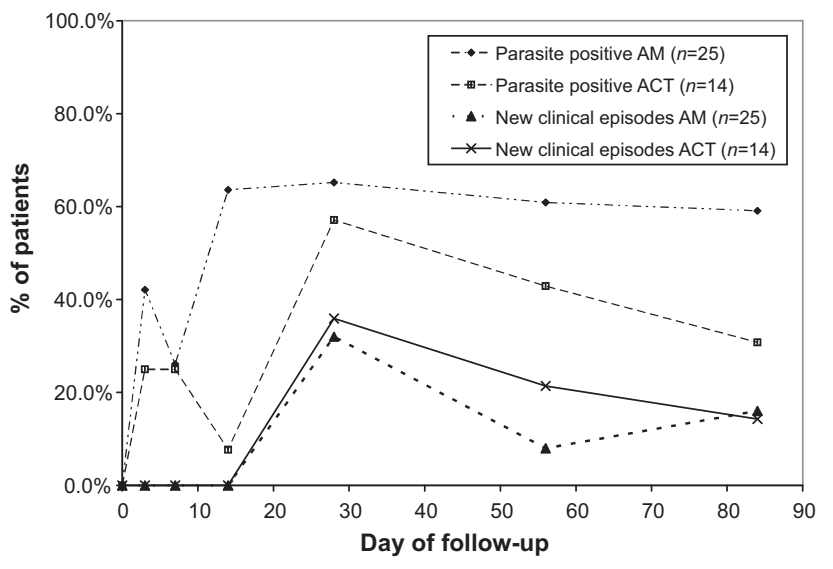

Figure 4. Parasite prevalence and new clinical episodes of malaria in patients with a negative malaria film at baseline. $(\mathrm{AM}=$ Argemone mexicana group, $\mathrm{ACT}=$ Artemisinin Combination Therapy group). The 'parasite positive' lines show the percentage of patients who had parasites on thick blood films at the chosen timepoints. The 'new clinical episodes' lines show the percentage of patients who experienced a new episode of clinical malaria since the last scheduled follow-up visit.

prevalence of moderate anaemia at day 84 (Table 3). There was no statistically significant difference in any outcome between those with or without parasites on the day 28 thick film, even after stratifying for age. There was a non-significant trend towards greater incidence of severe malaria and greater prevalence of anaemia in those who had no parasites at day 28. By logistic regression, the recurrence of malaria was found to be related to age (AOR 0.90 per added year of life, 95\% CI 0.87-0.94) but not to treatment group or day 28 parasitaemia (see Table 4 ).

\subsection{Adverse events}

Most adverse effects were short-term and have been discussed in detail elsewhere. ${ }^{7}$ Adverse events over the 84 day follow-up were noted, but most were probably unrelated to the treatment (for example, otitis media, urinary infections, sexually transmitted infections, malnutrition).

There were three serious adverse events in the course of the follow-up, two deaths in the AM group and one miscarriage in the ACT group. One of the deaths was probably due to severe malaria (see above). The other death (an 18-month old boy who died at day 35) was probably due to septicaemia. His initial illness was successfully treated on AM. At day 28 he complained of slight vomiting and fever the previous night but his axillary temperature was $35.5^{\circ} \mathrm{C}$ and his thick film was negative for malaria parasites. His parents were advised to return if there was any deterioration. At day 35 , he returned seriously ill in a coma (Blantyre score 1), with convulsions, jaundice, and anuria for 24 hours. His blood glucose was $3.9 \mathrm{mmol} / 1$, his haematocrit was $25 \%$, and on the thick film there were very few malaria parasites (41 per $\mathrm{mcl}$ ), but numerous neutrophils. He was given intramuscular artemether and cefotaxime and referred urgently to hospital, but died before arriving there. In view of the very low parasitaemia and high neutrophilia, the fatal illness was most probably septicaemia.

One miscarriage at about 13 weeks of gestation was observed in a woman in the ACT group. At the start of treatment she was about 8 weeks pregnant, and was mistakenly treated with As/Aq instead of SP. At day 35 after treatment she experienced a miscarriage with profuse bleeding, needing referral to hospital for evacuation of retained products of conception.

\subsection{Pregnant women}

Nineteen women were pregnant at the start of the study, 13 in the AM group and six in the ACT group. Seven of these had negative blood films, and only three had $>100$ parasites per mcl at baseline. A further two women in the AM group became pregnant during the course of follow-up. Twenty

Table 3

Outcome measures over months 2 and 3 (days 29-84) according to the presence of malaria parasites in a thick film at day 28 [95\% CI].

\begin{tabular}{|c|c|c|c|}
\hline & Parasites present at day $28(n=201)$ & Parasites absent at day $28(n=87)$ & $P$-value \\
\hline $\begin{array}{l}\text { Incidence per patient of uncomplicated } \\
\text { malaria (confirmed) }\end{array}$ & $0.34(68 / 201)[0.27-0.41]$ & $0.27(23 / 86)[0.18-0.37]$ & 0.46 \\
\hline $\begin{array}{l}\text { Age-specific incidence of severe malaria, in } \\
\text { patients aged } \leq 5 \text { years }\end{array}$ & $3 / 112(2.7 \%)[0.6-7.6]$ & $3 / 38(7.9 \%)[1.7-21.4]$ & 0.33 \\
\hline $\begin{array}{l}\text { Prevalence of moderate anaemia at day } 84, n \\
(\%)\end{array}$ & $1 / 190(0.5 \%)[0.0-2.9]$ & $2 / 81(2.5 \%)[0.3-8.6]$ & 0.36 \\
\hline
\end{tabular}

Table 4

Logistic regression model of factors influencing the recurrence of clinical episodes of malaria at days 29-84.

\begin{tabular}{lll}
\hline Variable & Regression coefficient & P-value \\
\hline Treatment Group = AM (ref = ACT) & 1.107 & $0.527-2.322$ \\
Sex = F (ref = M) & 1.162 & $0.616-2.193$ \\
Age & 0.897 & $0.852-0.944$ \\
Body temperature at day 29 & 0.734 & $0.450-1.199$ \\
Haematocrit at day 28 & 0.972 & 0.000 \\
Parasitaemia at day 28 $=$ & Ref & 0.217 \\
$1-99$ & 0.655 & $0.4601-1.049$ \\
$100-1999$ & 0.682 & $0.282-1.520$ \\
$2000-15000$ & 1.246 & $0.266-1.750$ \\
$>15000$ & 1.334 & $0.413-3.761$ \\
\end{tabular}

AM: Argemone mexicana group; ACT: Artemisinin Combination Therapy group. 
of the 21 women were followed up seven months after the start of the study, of whom seven (35\%) were still pregnant.

The babies resulting from the normal deliveries were all examined and found to be healthy. One miscarriage was reported as a serious adverse event at day 35 after treatment in the ACT group (see section on adverse events, above). Three miscarriages occurred in the AM group after the end of the $84 \mathrm{~d}$ follow-up, two of them in women who only discovered their pregnancies at days 72 and 84, so were not pregnant when they took AM. One stillbirth occurred five months after the treatment with AM.

\subsection{Need for re-treatment and cost}

Between days 29-84, re-treatment with the first-line antimalarial was needed at least once by $38.3 \%$ and $42.6 \%$ of patients in the AM and ACT groups respectively $(P=0.48)$. Over the same period, at least one treatment with the second-line antimalarial was required by $10.9 \%$ in the AM group compared to $5.0 \%$ in the ACT group $(P=0.08)$. Nevertheless $81 \%$ of patients in the AM group completed the 84 day follow-up without needing treatment with an ACT. This compares with all patients in the ACT group receiving the first line ACT, and a further $10 \%$ needing the second-line ACT. The average overall malaria-related treatment costs over the full 84 days of follow-up were 310 CFA Francs (FCFA) per patient in the AM group and 1338 FCFA per patient in the ACT group ( 1 Euro $=655$ FCFA; $P<0.000001$ ).

\section{Discussion}

\subsection{Principal findings}

Over all the 84 days of follow-up, there were no significant differences between the treatment groups in the incidence of uncomplicated malaria, severe malaria, moderate anaemia, or serious adverse events. Exploratory analyses showed that, in this context of high malaria transmission, total parasite clearance at d 28 was not associated with a reduced incidence of uncomplicated or severe malaria or of moderate anaemia over the subsequent two months. In the AM group $81 \%$ of patients completed the 84 days of follow-up without needing treatment with an ACT, and the cost per patient was a quarter of the cost in the ACT group.

\subsection{Strengths and weaknesses of the study}

This is the first study to our knowledge which examines whether total parasite clearance at day 28 is clinically important in a context of high malaria transmission. The randomisation worked well, as evidenced by the very similar baseline characteristics. We did not test for helminth infections which might have influenced some of the outcome measures including treatment response and incidence of anaemia. This is the largest randomised controlled trial of any herbal remedy for malaria, with the longest follow-up, and the first to include young children (who are at greatest risk, and therefore in greatest need of treatment).
We kept samples of each batch of plant material for further analysis, but at the time of the study the compound(s) responsible for the antimalarial activity in vivo had not been identified with certainty, so we could not standardise according to levels of 'active ingredients'. Nevertheless, the plants were all harvested from the same area of Mali so there was probably no great genetic or environmental heterogeneity which could have affected the levels of phytochemicals. Although we provided all patients with the same amount of plant material and gave the same instructions on the preparation of the decoction, we were not able to ensure that the preparation and dosage of the decoction was identical between patients and between episodes for the same patient. In a previous study we did provide a standard decoction (prepared by the traditional healer) to all patients ${ }^{6}$ but we felt that this would not be a practical public health intervention. In the present study we improved the practicality of the intervention at the expense of a level of standardisation. If the herbal decoction were to be used in public health programmes we felt it should be sufficiently safe and effective in spite of a level of variability in preparation and dosage which would occur in practice outside of the research setting.

There is no universally accepted method to confirm total parasite clearance. PCR would be the most sensitive but we did not have the resources to do this. We counted a microscopic examination as positive if at least one microscopist had seen a single parasite. This method is sensitive but runs the risk of having some false positives (artefacts may occasionally be mistaken for parasites). An alternative would be to count a slide as positive only if both microscopists saw at least one parasite. When we analysed the data using this less sensitive definition, the difference in incidence of uncomplicated malaria on d 29-84 between the parasite positive and negative at day 28 became significant ( $0.38 \mathrm{vs}$ 0.25 per patient, $P=0.02$ ) but there was still no difference in the incidence of severe malaria or anaemia. The absolute parasitaemia had no effect on the risk of recurrence of malaria in a logistic regression model, after adjusting for age.

The difference in outcome between those who took AM for one week and two weeks probably reflects underlying differences in the disease and patient immune levels, as patients were only advised to continue for $>7$ days if they still had persisting mild symptoms. We cannot draw any conclusions about the relative effectiveness of different lengths of treatment as patients were not allocated randomly to these. In the course of follow-up a small number of patients in each group received treatments with possible antimalarial effects, some modern (such as doxycyline, cotrimoxazole) and some traditional (such as Guiera senegalensis). However the proportion of patients taking these was less than $10 \%$ and equal in each group. Those who specifically took an antimalarial not prescribed by the study team were excluded from the analysis. Bednets were not widely used at the time of the study, although after the study ended we distributed bednets to all participants and families in the villages.

Five patients of the 102 new episodes in the AM group did not have a confirmatory blood film taken. If all of these were positive with a parasitaemia $>100 / \mathrm{mcl}$, the incidence 
of uncomplicated malaria in the AM group would have been $68 / 193=0.35$ per patient (instead of 0.33 ), but this still would not have been statistically significant $(P=0.61)$. If the true difference in incidence of severe malaria between the groups was $4.9 \%$ vs $1.9 \%$, this study would not have sufficient power to detect it as statistically significant. However with our sample size, we can be $95 \%$ confident that the agespecific incidence of severe malaria would remain under $6 \%$ per month (over months $2-3$ ) for children aged $\leq 5$ years treated with either AM or ACT, in similar conditions, which is lower than in comparable studies of HMM (see section on Meaning of the study, below).

The number of patients in this study was not large enough to detect rare adverse events with any certainty, and a larger study is needed in this regard. It would also have been ideal to follow up all the pregnant women at least to the end of their pregnancy.

\subsection{Meaning of the study: possible explanations and implications for clinicians and policymakers}

The finding that parasite clearance did not influence subsequent incidence of recurrent malaria or anaemia may be surprising to some. It has often been argued that residual or recurrent parasitaemia, even if asymptomatic, would lead to more episodes of malaria and more anaemia. Olliaro et al. ${ }^{11}$ found that recurrent asymptomatic parasitaemia (after initial clearance) progressed to fever in $42 \%$ of children, and argued that even asymptomatic recurrence of parasitaemia should be treated. Parasite clearance maintained up to day 28 is the gold standard outcome measure for antimalarial drug efficacy trials. ${ }^{9,12}$ Yet the clinical importance of total parasite clearance depends on the epidemiological context. In a low-transmission area where patients have little or no immunity, parasite clearance is clearly the goal. However in high-transmission areas where patients are semi-immune, and re-infection happens rapidly, total parasite clearance may not be clinically important. ${ }^{12}$ Asymptomatic parasitaemia (especially multiclonal) may even be beneficial in preventing clinical attacks of malaria. ${ }^{13,14}$ This study is the first to look at the consequences of persisting asymptomatic parasitaemia (rather than recurrence after initial clearance).

The most comparable study of HMM in a neighbouring area of Burkina $\mathrm{Faso}^{8}$ found an incidence of 'fever + coma/convulsions' of $5 \%$ per month for children receiving chloroquine, and $11 \%$ per month for those not receiving it. This is a narrower definition of severe malaria than ours (which included persistent vomiting, respiratory distress and severe anaemia). If we count only the cases of severe malaria which fit their definition, the incidence in our study was lower (about $1 \%$ per month).

This suggests that both AM and As/Aq would be useful treatments for the HMM. In a setting where resources are limited, the relatively expensive ACTs could be reserved for children under five years who are at greatest risk of severe malaria and death, while AM could safely be used for older patients. A health policy to use AM as first line for semi-immune patients, e.g. those $>5$ years old in a high transmission area, may also help to reduce the spread of resistance to ACTs. Wargo et al. ${ }^{15}$ have shown that the development of resistant strains might in fact be slower when parasite clearance is not achieved: where subcurative chemotherapy was administered, the resistant clone was only partly released from competitive suppression and experienced a restriction in the size of its expansion after treatment.

\subsection{Unanswered questions and future research}

Operational research is needed on strategies taking advantage of the potential of AM. A first step in such research should be the analysis of batches of AM to be used, in order to verify that the content of known anti-plasmodial compounds is sufficient. The plant contains at least three alkaloids with strong in vitro activity against $P$. falciparum: berberine, protopine and allocryptopine, ${ }^{16}$ but it remains to be seen if these are also active in vivo. Once the substances responsible for the clinical activity of AM have been identified, a simple test needs to be developed to measure their presence in various cultivars, and thus to control the quality of different plant batches.

It is possible that if bednets were widely available and used, the transmission of malaria might be reduced to the point where parasite clearance might become relevant to clinical outcomes. This would need to be evaluated in future studies. Furthermore better outcome measures need to be developed which distinguish between the issues of Plasmodium infection and malarial disease in areas where the prevalence of infection is high even among healthy individuals. The ultimate aim of malaria treatment is to prevent serious illness and death. In this regard more relevant outcome measures are incidence of severe malaria and mortality. Larger sample sizes would be needed to demonstrate relevant differences in these.

\section{Conclusions}

At the end of three months of follow-up in a high transmission area there was no difference in incidence of uncomplicated or severe malaria or anaemia between patients treated first-line with the locally produced AM decoction and patients treated with As/Aq, or between patients with and without total parasite clearance at day 28. The incidence of severe malaria was kept to a lower level than reported in other studies of HMM. If these findings can be confirmed, some antimalarials which are clinically effective but do not produce parasite clearance could nevertheless be appropriate in high transmission areas. Thus AM could be tested operationally as first-line treatment of uncomplicated malaria in semi-immune patients (patients $>5$ years old) in high transmission areas, and as first aid when modern antimalarials are not immediately accessible. Such a policy could be tested as a way to delay resistance to ACTs.

Authors' contributions: MLW, BG, CD, JF and DD designed the study protocol; MLW, OS and FD carried out the clinical assessment, laboratory analyses and follow-up. All authors worked on analysis and interpretation of the data. MLW drafted the manuscript, and all authors read and approved the final manuscript. MLW is guarantor of the paper. 
Acknowledgements: We wish to thank the inhabitants of Missidougou, in particular village chief Tiemoko Bengaly and his son, the village health worker, Madou Bengaly, without whose open and enthusiastic collaboration this study would not have been possible. We are also very grateful to our fieldwork staff, Mr Fagnan Sanogo, Mr Diafara Berthé, Mr Yussuf Berthé, and Dr Modibo Doumbia for their hard work in the field. We would like to thank the staff and doctoral students of the Département de Médecine Traditionnelle, the Malian health authorities, the National Malaria Control Programme, the PSDS office in Sikasso, the Médecins Sans Frontières Mali office, Blaise Genton, Bernard Burnand, Jean-Pierre Gervasoni (Lausanne) and Jean-Paul Guthmann at Epicentre (Paris) for their invaluable advice in the planning and analysis of this study. We thank Dick Mayon-White and Hubert Barennes for helpful comments on the article.

Funding: Swiss Agency for Development and Cooperation (SDC).

Conflicts of interest: None.

Ethical approval: Ethics Committee of the 'Institut National de Recherche en Sante Publique' (INRSP) of Mali, 6 June 2006.

Note: When the study was being planned, there was no registration number in the ethical clearance system for the INRSP Ethics Committee in Bamako.

\section{References}

1. D’Alessandro U, Talisuna A, Boelaert M. Should artemisinin-based combination treatment be used in the home-based management of malaria? Trop Med Int Health 2005;10:1-2.

2. Willcox ML, Bodeker G. Traditional herbal medicines for malaria. BMJ 2004;329:1156-9.

3. Diallo D, Graz B, Falquet J, Traore AK, Giani S, Mounkoro PP, et al. Malaria treatment in remote areas of Mali: use of modern and traditional medicines, patient outcome. Trans $R$ Soc Trop Med Hyg 2006;100:515-20.
4. Graz B, Diallo D, Falquet J, Willcox M, Giani S. Screening of traditional herbal medicine: first, do a retrospective study, with correlation between diverse treatments used and reported patient outcome. J Ethnopharmacol 2005;101:338-9.

5. Diallo D, Diakite C, Mounkoro PP, Sangare D, Graz B, Falquet J, et al. Knowledge of traditional healers on malaria in Kendi (Bandiagara) and Finkolo (Sikasso) [in Mali]. Mali Med 2007;22:1-8.

6. Willcox ML, Graz B, Falquet J, Sidibe O, Forster M, Diallo D. Argemone mexicana decoction for the treatment of uncomplicated falciparum malaria. Trans R Soc Trop Med Hyg 2007;101:1190-8.

7. Graz B, Willcox ML, Diakite C, Falquet J, Dackuo F, Sidibe O, et al. Argemone mexicana decoction versus artesunate-amodiaquine for the management of malaria in Mali: policy and public-health implications. Trans R Soc Trop Med Hyg 2010;104:33-41.

8. Sirima SB, Konate A, Tiono AB, Convelbo N, Cousens S, Pagnoni F. Early treatment of childhood fevers with pre-packaged antimalarial drugs in the home reduces severe malaria morbidity in Burkina Faso. Trop Med Int Health 2003;8:133-9.

9. WHO. Assessment and monitoring of antimalarial drug efficacy for the treatment of uncomplicated Falciparum malaria. Geneva: World Health Organization; 2003.

10. Coulibaly D, Diallo DA, Thera MA, Dicko A, Guindo AB, Kone AK, et al. Impact of preseason treatment on incidence of falciparum malaria and parasite density at a site for testing malaria vaccines in Bandiagara, Mali. Am J Trop Med Hyg 2002;67:604-10.

11. Olliaro P, Pinoges L, Checchi F, Vaillant M, Guthmann JP. Risk associated with asymptomatic parasitaemia occurring post-antimalarial treatment. Trop Med Int Health 2008;13:83-90.

12. Staedke SG, Mwebaza N, Kamya MR, Clark TD, Dorsey G, Rosenthal PJ, et al. Home management of malaria with artemetherlumefantrine compared with standard care in urban Ugandan children: a randomised controlled trial. Lancet 2009;373: 1623-31.

13. Farnert A, Rooth I, Svensson, Snounou G, Bjorkman A. Complexity of Plasmodium falciparum infections is consistent over time and protects against clinical disease in Tanzanian children. J Infect Dis 1999;179:989-95.

14. Males S, Gaye O, Garcia A. Long-term asymptomatic carriage of Plasmodium falciparum protects from malaria attacks: a prospective study among Senegalese children. Clin Infect Dis 2008;46: 516-22.

15. Wargo AR, Huijben S, de Roode JC, Shepherd J, Read AF. Competitive release and facilitation of drug-resistant parasites after therapeutic chemotherapy in a rodent malaria model. Proc Natl Acad Sci USA 2007; 104:19914-9.

16. Simoes-Pires CA. Investigation of antiplasmodial compounds from various plant extracts: Argemone mexicana L. (Papaveraceae), Licania octandra (Hoffmanns. ex. Roem \& Schult) Kuntze (Chrysobalanaceae) and Syzygium cumini (L.) Skeels (Myrtaceae). Geneva: Universite de Geneve; 2009. 